1.5 seconds is not a constant-current machine, and in a recent article by Pippard (Journal, May 1992, 160, 621-637) a stimulus length of 1.5 seconds does not appear to be available from constant-current ECT machinery. I wonder if the stimulus dosage given is actually known at all. Could more data about the stimulus dosage given at ECT offer an explanation? The complex relationship between stimulus dosing and seizure duration may offer an explanation. The first course of ECT in their patient may have produced no therapeutic effect despite producing convulsions because the dosage did not exceed the seizure threshold adequately for therapeutic effect. There could with time, or the waning benzodiazepine effect, have been a change in seizure threshold in their patient. I think it is about time that ECT was not regarded as a black hole and that the dosage received by patients was adequately charted for scientific study of its effect.

RusselL, R. J. (1988) Stimuli used in ECT. In Current Approaches to ECT (eds J. C. Malkin \& S. Brandon), pp. 58-60. Southampton: Duphar Medical Relations.

Wonford House Hospital

R. F. HILL

Dryden Road

Exeter EX2 5AF

AUTHORs' REPLY: Dr Hill is quite right that the dosage of ECT received by patients should be known and recorded, and I am afraid that we cannot be as precise as he would like. However, the essential point we make is that the same technique with the same machine was used on each occasion, and that the presence of benzodiazepines appeared to have prevented the response to ECT. We are not sufficiently expert to comment on the exact reason for this, but the lesson is that these drugs must be discontinued some time before giving ECT to depressed patients.

Samuel. I. Cohen

The Royal London Hospital Trust Claire lawton

St Clement's

$2 A$ Bow Road

London E3 4LL

\section{ECT for young people}

SIR: There has been recent controversy about the use of electroconvulsive therapy (ECT) to treat young people. Consequently, we reviewed the frequency of, and the indications for, the use of ECT in patients under 18 years of age treated at the Royal Edinburgh Hospital over the past ten years. The hospital is a psychiatric teaching hospital that serves a population of $\mathbf{4 4 0 0 0 0}$ and is the only site in Edinburgh in which ECT could be given to young people. Written ECT records are still available from January 1982 and follow-up of young people treated by ECT was possible by case-note review up to June 1992

Five people under 18 years were treated with ECT. All were female and all were 17 years old at the time. Patient 1 was treated in 1982 for a delusional depressive illness that had not responded to a tricyclic antidepressant alone or in combination with a neuroleptic drug. Subsequently, the patient had 17 admissions to the hospital and the diagnosis was revised to one of schizophrenia. Patient 2 was treated in 1986 for the depressive phase of a manicdepressive illness that had not responded to a tricyclic antidepressant alone or in combination with lithium carbonate. Patient 3 was treated in 1987 for depressed mood associated with delusions, but further details were unavailable because of the loss of the relevant casenotes. The patient remained in contact with the psychiatric services and the diagnosis was revised to one of schizophrenia. Patient 4 was treated in 1990 for a post-partum delusional depressive illness that had not responded to two different tricyclic antidepressants. Patient 5 was treated in 1991 for a delusional depressive illness that had not responded to two different tricyclic antidepressants. The diagnosis was revised to one of schizophrenia during follow-up.

The average frequency of the use of ECT in people under 18 years in Edinburgh was one case every two years. The indications were always depression associated either with psychotic features or manic-depressive illness.

The Royal College of Psychiatrists has not published guidelines for the use of ECT in young people, although the ECT Sub-Committee is presently revising the guidelines on the practical administration of ECT. The American Psychiatric Association Task Force on ECT (1990) recommended that ECT should never be used as a primary treatment for children or adolescents. ECT was recommended where the index illness had not responded to treatment, when there was a need for a rapid and definitive improvement in the illness for either medical or psychiatric reasons, or where ECT was likely to produce less severe adverse effects. There was no evidence that ECT was used inappropriately in the present review based on these APA recommendations. The APA also recommends that an independent psychiatrist experienced in treating adolescents reviews the indication for ECT, and this would be one area of practice that falls short of the recommendations. 
The APA also noted that appropriate procedures for consent had not yet been agreed and that further research on the indications, benefits and adverse effects of ECT in young people was required. We do not know if the present findings can be generalised to the whole of the UK, but we found no evidence for the inappropriate use of ECT in young people over the past ten years in Edinburgh.

American Psychiatric Association Task Force on ECT (1990)

The practice of ECT: recommendations for treatment, training and privileging. Convulsive Therapy, 6, 85-120.

Alastair CoOK Allan SCOTT

Royal Edinburgh Hospital

Morningside Terrace

Edinburgh EHIO 5HF

\section{Community care and suicide}

SIR: Boer \& Briscoe (Journal, June 1992, 160, 867) state that their analysis of overall rates of suicide and open verdicts in three West-country health districts has not revealed evidence that a change of emphasis towards community care is associated with demonstrable effects on suicide rates.

The previous inquiry based in one of these health districts (Journal, February 1992, 160, 149-153) indicated that the raw numbers of suicides were too small to allow firm conclusions to be drawn from them. Even though Drs Boer and Briscoe amalgamated three districts in their data analysis, the numbers of suicides and open verdicts must surely have remained small, although they give no details. Such statistical problems, related to the low base rate of suicide, mean that the overall rate of suicides and open verdicts based on small populations inevitably remains an inadequate index of competence of service provision.

A more sensitive measure of the effectiveness of a mental-health service is the rate of suicides and open verdicts in those persons who have made direct contact with its various components. We believe it is important that each discrete service should set up a register which is able to monitor such data in a reliable way, including both community and hospital-style facilities. In this way, trends in suicides and open verdicts can be identified, as well as foci of particular difficulty which may need attention. Such a register can be set up relatively easily without requirement of extra administrative staff: a recent study has demonstrated that information about suicides and open verdicts in Avon, wherever they have died in the UK, was readily available.

Regular clinical multidisciplinary audit of unexpected deaths in persons who have recently been in contact with psychiatric services is therefore feasible. and we believe essential, in evaluating the effectiveness of service provision. This is particularly so at the present time of rapid change in service organisation.

H. G. MORGAN

University of Bristol

C. A. Vassilas

Department of Mental Health

41 St Michael's Hill

Bristol BS2 8DZ

\section{5-HT 2 receptors, hallucinations, and dementia}

SIR: I read with interest Haddad \& Benbow's article (Journal, August 1992, 161, 263-265). I would like to comment on the strong association between the dementing processes and isolated visual hallucinations as indicated by the authors.

A number of investigators have hypothesised that hallucinogens act as direct agonists at the 5-hydroxytryptamine ${ }_{2}\left(5-\mathrm{HT}_{2}\right)$ receptor in the central nervous system (for review see Pierce \& Peroutka, 1990). The affinity of hallucinogenic agents for 5- $\mathrm{HT}_{2}$ binding sites correlates significantly with their potencies for causing hallucinations in humans (Glennon et al, 1984) and results in a rapid desensitisation and downregulation of the central 5- $\mathrm{HT}_{2}$ receptors in humans and laboratory animals (Leysen \& Pauwels, 1991).

Downregulation of $5-\mathrm{HT}_{2}$ receptors has also been reported consistently during the dementing processes (Cross et al, 1986; Gross-Iseroff et al, 1990).

The initial rapid desensitization and down regulation of 5- $\mathrm{HT}_{2}$ receptors may therefore explain the early presence of hallucinations during the dementing processes.

Cross, A. J., Crow, T. J., Ferrier, I. N., et al (1986) The selectivity of the reduction of serotonin S2 receptors in Alzheimer-type dementia. Neurobiology of Aging, 7, 3-7.

Glennon, R. A., Titeler, M. \& MCKenney, J. D. (1984) Evidence for 5-HT, involvement in the mechanism of action of hallucinogenic agents. Life Sciences, 35, 2505-2511.

Gross-Isseroff, R., SAlama, D., ISRAEL, M. et al (1990) Autoradiographic analysis of age-dependent changes in serotonin S-HT, receptors of the human brain postmortem. Brain Research, 519, 223-227.

LeYsen, J. E. \& PAUWELS, P. J. (1990) 5-HT, receptors, roles and regulation. Annals of the New York Academy of Sciences, 600 , 183-193.

Pierce, A. P. \& PeroutKa, S. J. (1990) Antagonist properties of d-LSD at 5 -hydroxytryptamine, receptors. Neuropsychopharmacology, 3, 503-508.

Serdar M. Dursun

Pharmaceutical Sciences Institute

Aston University

Aston Triangle

Birmingham B4 7ET 